\title{
Automatic Ventral Intermediate Nucleus Localization Based on Anterior Commissure and Posterior Commissure
}

\author{
Riyanarto Sarno ${ }^{1 *} \cdot$ Kelly Rossa Sungkono ${ }^{1} \bullet$ Mohammad Ardika Rifqi ${ }^{1}$ \\ Achmad Fahmi ${ }^{2,3}$ • Agus Turchan ${ }^{2,3}$ Abdul Hafid Bajamal ${ }^{2,3}$ \\ *Corresponding author’s Email: riyanarto@if.its.ac.id \\ ${ }^{1}$ Department of Informatics, Institut Teknologi Sepuluh Nopember Surabaya, Indonesia \\ ${ }^{2}$ Department of Neurosurgery, Faculty of Medicine, Airlangga University, Indonesia \\ ${ }^{3}$ Dr. Soetomo General Academic Hospital, Medical Center. Indonesia
}

\begin{abstract}
The ventral intermediate nucleus (Vim) of the thalamus is a brain lesion target for decreasing tremors in Parkinson's disease patients. Determination of the location of the Vim is challenging because magnetic resonance imaging (MRI) cannot detect it. Several Vim targeting methods have been proposed, such as Coordinate-based targeting and Guiot's methods. However, these methods are manually implemented by doctors based on MRI data, so the accuracy of the targeting depends on their memory and foresight. This paper proposes a method to automate Vim localization using Coordinate-based targeting and Guiot's method. Firstly, this paper determines the plane of the anterior commissure (AC) and the posterior commissure (PC) because to enforce medical images and determine the centre as the middle of AC-PC. Secondly, the coordinates are converted from millimetres to voxels in determining the Vim location. The converter is needed because Coordinate-based targeting and Guiot's method measure the Vim location in millimetres while the three-dimensional images are measured in voxels. This paper also calculates similarity percentages and averages of error results to determine the accuracy and the robustness of automatic Coordinate-based targeting and Guiot's. The experiments verify Coordinate-based targeting is higher accuracy and more robust than Guiot's.
\end{abstract}

Keywords: brain lesion, comparison experiment, magnetic resonance imaging, Parkinson's disease, ventral intermediate nucleus.

\section{INTRODUCTION}

Parkinson's disease is a general neurological disorder that mostly afflicts elderly people [1]. Several studies have been conducted on treating Parkinson's by brain lesion [2-4]. The ventral intermediate nucleus (Vim) is a brain lesion target for Parkinson's disease to decrease tremors $[3,5,6]$. Long-term clinical patient outcomes, with a follow up of at least 5 years, have shown no tremors in the contralateral limbs. Moreover, contralateral rigidity improved in most of the patients [7]. However, the Vim cannot be identified with certainty, even by using 7 Tesla magnetic resonance imaging (MRI) [4, 8].

To deal with this problem, several methods, such as Guiot's method $[9,10]$ and Coordinatebased targeting $[4,11]$, have been developed to identify the location of the Vim. Coordinate-based targeting determines the coordinates of the Vim based on the human brain atlas data, i.e. SchatelbrandBailey's atlas [12]. Guiot's method determines the y-coordinate of the Vim based on the real distance between the anterior commissure (AC) and the posterior commissure (PC) of the patient and the $\mathrm{x}$ coordinate based on the Coordinate-based targeting method. Several neurosurgical planning applications are available, such as [12-15]. All applications provide a three-dimensional view of the MRI; however, they cannot perform automatic Vim targeting. These applications need the memory and the foresight of a doctor to accurately determine the Vim location.

This paper proposes an automatic Vim localization method based on the Coordinate-based targeting method and Guiot's method. The first step of the proposed method is determining the plane of the anterior commissure (AC) and the posterior commissure (PC) to enforce medical images and determine the centre as the middle of AC-PC. Secondly, the coordinates are converted from millimetres to voxels in determining the Vim location. The converter is needed because both of Coordinate-based targeting and Guiot's measure the Vim location in millimetres while the three-dimensional MRI are measured in voxels. The goal of the proposed method is Vim location based on automatic Coordinatebased targeting and Guiot's.

The experiment of this paper will determine the correctness of the proposed method for automatic Vim localization by comparing the results of the proposed method and manual method that 
is implemented in an existing application, Inomed Planning Software [16]. This paper also measures the accuracy and verifies the robustness of automatic Coordinate-based and Guiot's. The accuracy is obtained by similarity percentages between the results of the methods and the ground truth by using Euclidean Distance. The robustness utilizes the average of error results. A method is more robust if the average error results are smaller than the others.

\section{RELATED METHODS}

2.1. Vim

The ventral intermediate nucleus (Vim) is commonly used in the surgical treatment of Parkinsonian and essential tremors [17]. The Vim is a nuclear part of the thalamus, which delivers signals from sensors, such as motor signals. The location of the Vim cannot be identified with certainty, even when using 7 Tesla magnetic resonance imaging. However, it can be detected by referring to surrounding parts of a brain. The Vim is located approximately $1.5 \mathrm{~mm}$ next to the corticospinal tract (CST), which can be observed in MRI (shown in the red circle in Figure 1).

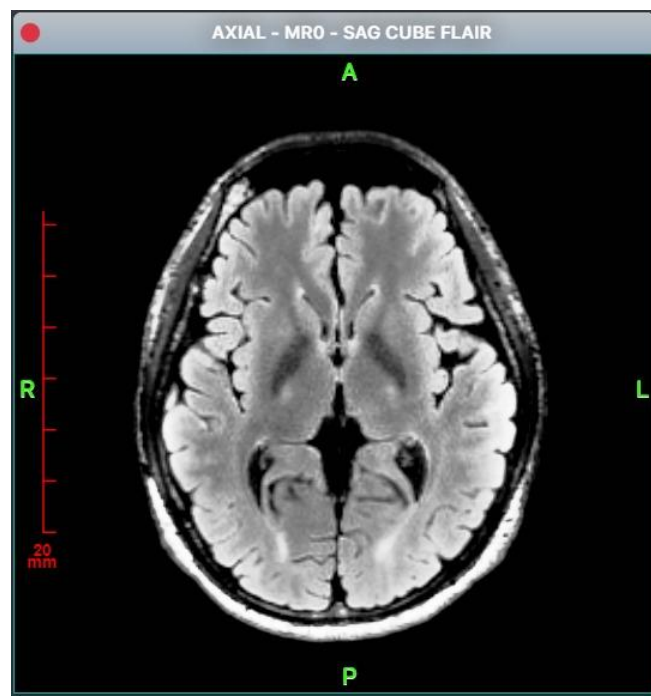

Figure 1. Axial plane in brain magnetic resonance imaging (MRI)

\subsection{Methods of Vim Targeting}

There are two general methods for finding the location of Vim, i.e. Coordinate-based targeting and Guiot's method. Coordinate-based targeting $[4,11]$ is a Vim localization method based on the human brain atlas data, i.e. Schatelbrand-Bailey's atlas. The equations for locating the Vim are given in Theorem 1, whose symbols are explained in Definition 1.

Definition 1. $A C$ is the point of the anterior commissure and $P C$ is the point of the posterior commissure in the anterior commissure-posterior commissure plane; $\left(x_{A C}, y_{A C}\right)$ are the $x$-coordinate and the $y$ coordinate of the anterior commissure; $\left(x_{P C}, y_{P C}\right)$ are the $x$-coordinate and the $y$-coordinate of the posterior commissure; $\left(x_{V M_{M_{R}}}, y_{V M_{M_{R}}}\right)$ are the $x$-coordinate and the $y$-coordinate of the right Vim in the MRI based on the method; $\left(x_{V M_{u_{L}}}, y_{V M_{w_{L}}}\right)$ are the $x$-coordinate and the $y$-coordinate of the left Vim in MRI based on the method; $d(A C, P C)$ is the distance between the anterior commissure (AC) and the posterior commissure (PC).

\section{Theorems 1.}

$\left(x_{V I M_{M_{R}}}, y_{V I M_{M_{R}}}\right)=\left(x_{P C}-15 m m, y_{P C}+[6,7] \times d(A C, P C)\right)$ and $\left(x_{V I M_{M_{L}}}, y_{V I M_{M_{L}}}\right)=\left(x_{P C}+5 m m, y_{P C}+[6,7] \times d(A C, P C)\right)$ 
Guiot's method $[9,10]$ determines the y-coordinate of the Vim based on the real distance between the anterior commissure (AC) and the posterior commissure (PC) of the patient and the $\mathrm{x}$ coordinate based on the Coordinate-based targeting method. The equations for locating the Vim are given in Theorem 2, whose symbols are explained in Definition 1.

\section{Theorem 2.}

$$
\left(x_{V I M_{M_{R}}}, y_{V I M_{M_{R}}}\right)=\left(x_{P C}-15 m m, y_{P C}+\frac{1}{4} d(A C, P C) m m\right) \text { and }\left(x_{V M_{M_{L}}}, y_{V M_{M_{L}}}\right)=\left(x_{P C}+15 m m, y_{P C}+\frac{1}{4} d(A C, P C) m m\right)
$$

\subsection{Measurements of Accuracy and Robustness}

This paper measures the accuracy by using a similarity percentage and the robustness by using an average of error result. The similarity percentage in Equation (1) utilizes the division number between one with the addition of one and Euclidian Distance of Vim locations determined by a Vim targeting method and a ground truth. The ground truth is a Vim location of a brain lesion in a postoperative medical image. The average error result is determined in Equation (2). The used medical images for calculating the error result are MRI of patients with several slopes, i.e. $-10^{\circ},-5^{\circ}, 5^{\circ}, 10^{\circ}$ toward X-plane, $-10^{\circ},-5^{\circ}, 5^{\circ}, 10^{\circ}$ toward Y-plane, and $-10^{\circ},-5^{\circ}, 5^{\circ}, 10^{\circ}$ toward Z-plane.

$$
S P=\frac{1}{1+\left(\frac{\sum_{1}^{n} \sqrt{\left(x_{M_{n}}-x_{G_{n}}\right)^{2}+\left(y_{M_{n}}-y_{G_{n}}\right)^{2}+\left(z_{M_{n}}-z_{G_{n}}\right)^{2}}}{n}\right)} \times 100 \%
$$

where: $S P \quad:$ a similarity percentage

$n \quad:$ a number of patients

$x_{M_{n}} \quad$ : a X-coordinate of Vim location determined by a Vim targeting method

$x_{G_{n}} \quad$ : a X-coordinate of Vim location in a post-operative medical image as ground truth

$y_{M_{n}} \quad$ : a y-coordinate of Vim location determined by a Vim targeting method

$y_{G_{n}} \quad$ : a y-coordinate of Vim location in a post-operative medical image as ground truth

$Z_{M_{n}} \quad$ : a z-coordinate of Vim location determined by a Vim targeting method

$z_{G_{n}} \quad:$ a z-coordinate of Vim location in a post-operative medical image as ground truth

$A E R_{S}=\frac{\sum_{1}^{n} \sqrt{\left(x_{M_{S_{n}}}-x_{M_{0_{n}}}\right)^{2}+\left(y_{M_{S_{n}}}-y_{M_{0_{n}}}\right)^{2}+\left(z_{M_{S_{n}}}-z_{M_{0_{n}}}\right)^{2}}}{n}$

where: $A E R_{S}:$ an average of error results in a medical image with a certain slope

$n \quad:$ a number of patients

$x_{M_{S_{n}}} \quad:$ a x-coordinate of Vim location determined by a Vim targeting method in a medical image with a certain slope

$x_{M_{0_{n}}} \quad$ : a x-coordinate of Vim location determined by a Vim targeting method in a medical image with $0^{\circ}$ degree toward $\mathrm{x}, \mathrm{y}$, and $\mathrm{z}$ planes as ground truth

$y_{M_{S_{n}}} \quad$ : a y-coordinate of Vim location determined by a Vim targeting method in a medical image with a certain slope

$y_{M_{0_{n}}} \quad$ : a y-coordinate of Vim location determined by a Vim targeting method in a medical image with $0^{\circ}$ degree toward $\mathrm{x}, \mathrm{y}$, and $\mathrm{z}$ planes as ground truth

$z_{M_{S_{n}}} \quad$ : a z-coordinate of Vim location determined by a Vim targeting method in a medical image with a certain slope

$z_{M_{0}} \quad$ : a z-coordinate of Vim location determined by a Vim targeting method in a medical image with $0^{\circ}$ degree toward $\mathrm{x}, \mathrm{y}$, and $\mathrm{z}$ planes as ground truth 


\section{MATERIAL AND METHODS}

\subsection{Material}

This research used post-operative MRI T2 Flair images of twelve Parkinson's disease patients. The patients consisted of three women and eight men. The detail of patients as the data is shown in Table 1. One of the patients had a cyst related corpus callosum. Corpus callosum of patients with and without a cyst are denoted as red circles in Figure 2 and Figure 3. Because the cyst, the shape of corpus callosum (CC) in a patient with a cyst shrinks compared to a patient without a cyst. The small shape of $\mathrm{CC}$ complicates the determination of AC and PC, so the accuracy of the Vim location will decrease.

The data used in this paper were obtained after ethical clearance 1619/KEPK/XI/2019 from Dr. Soetomo General Academic Hospital. The MRI images were acquired from 3T MRI scanners. The Leksell Surgiplan application [13] was used to semi-automatically determine the Vim location during surgery. The post-operative MRI data were collected as input data.

Table 1. Details of Patients

\begin{tabular}{lll}
\hline \hline Patient & Sex (Female or Male) & $\begin{array}{l}\text { Length of } \boldsymbol{d}(\mathbf{A C}, \mathbf{P C}) \\
(\mathbf{m m})\end{array}$ \\
\hline \hline 1 & Male & 26.520 \\
\hline 2 & Male & 25.530 \\
\hline 3 & Female & 23.431 \\
\hline 4 & Female & 25.713 \\
\hline 5 & Male & 23.902 \\
\hline 6 & Male & 23.571 \\
\hline 8 & Male & 24.573 \\
\hline 9 & Male & 29.317 \\
\hline 10 & Female & 22.758 \\
\hline 11 & Female & 23.374 \\
\hline \hline (AC, PC) & Male & 23.892 \\
\hline
\end{tabular}

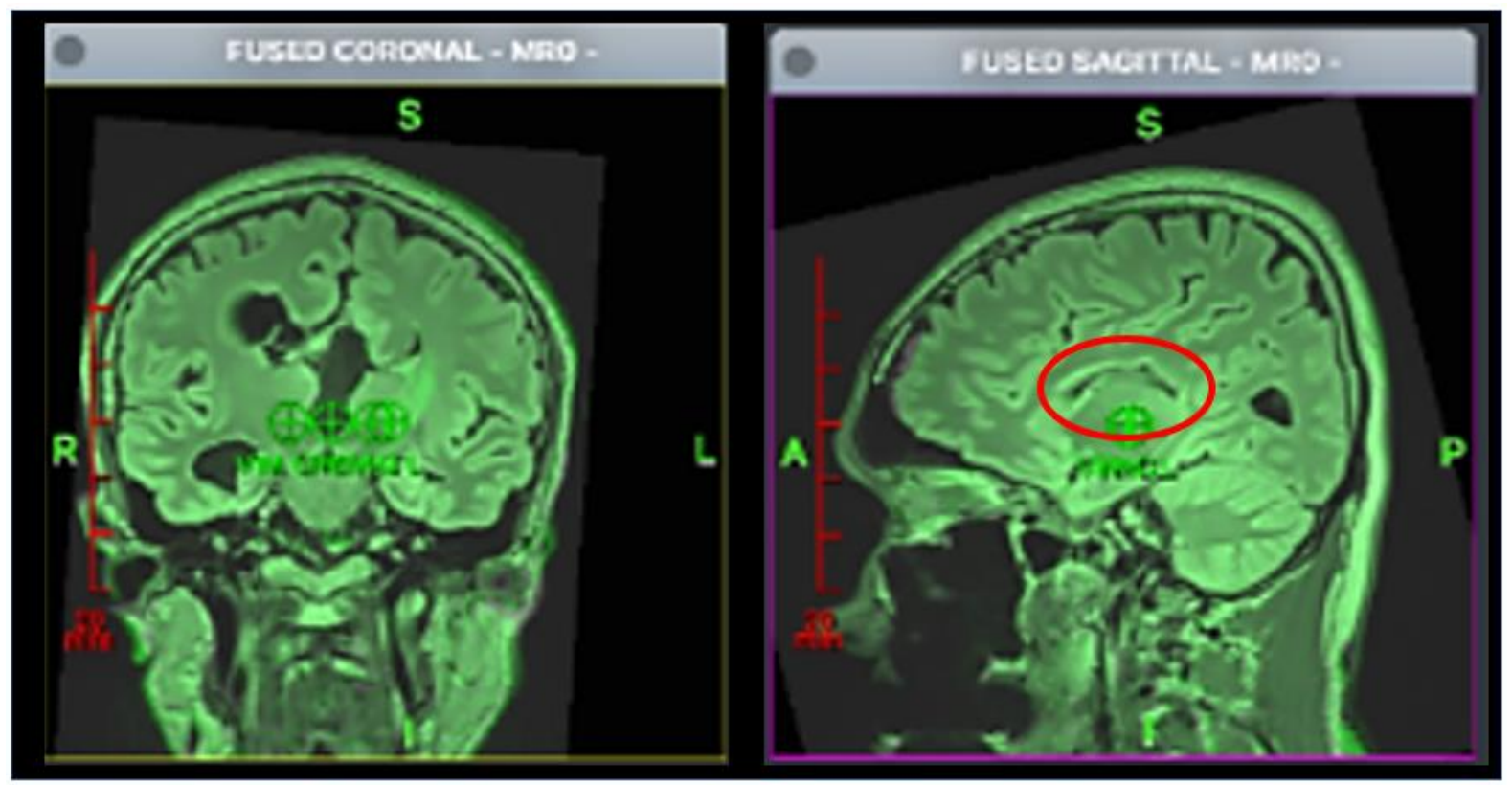

Figure 2. The example of Coronal and Axial Pslanes of Patients with Cyst (Patient 2). 


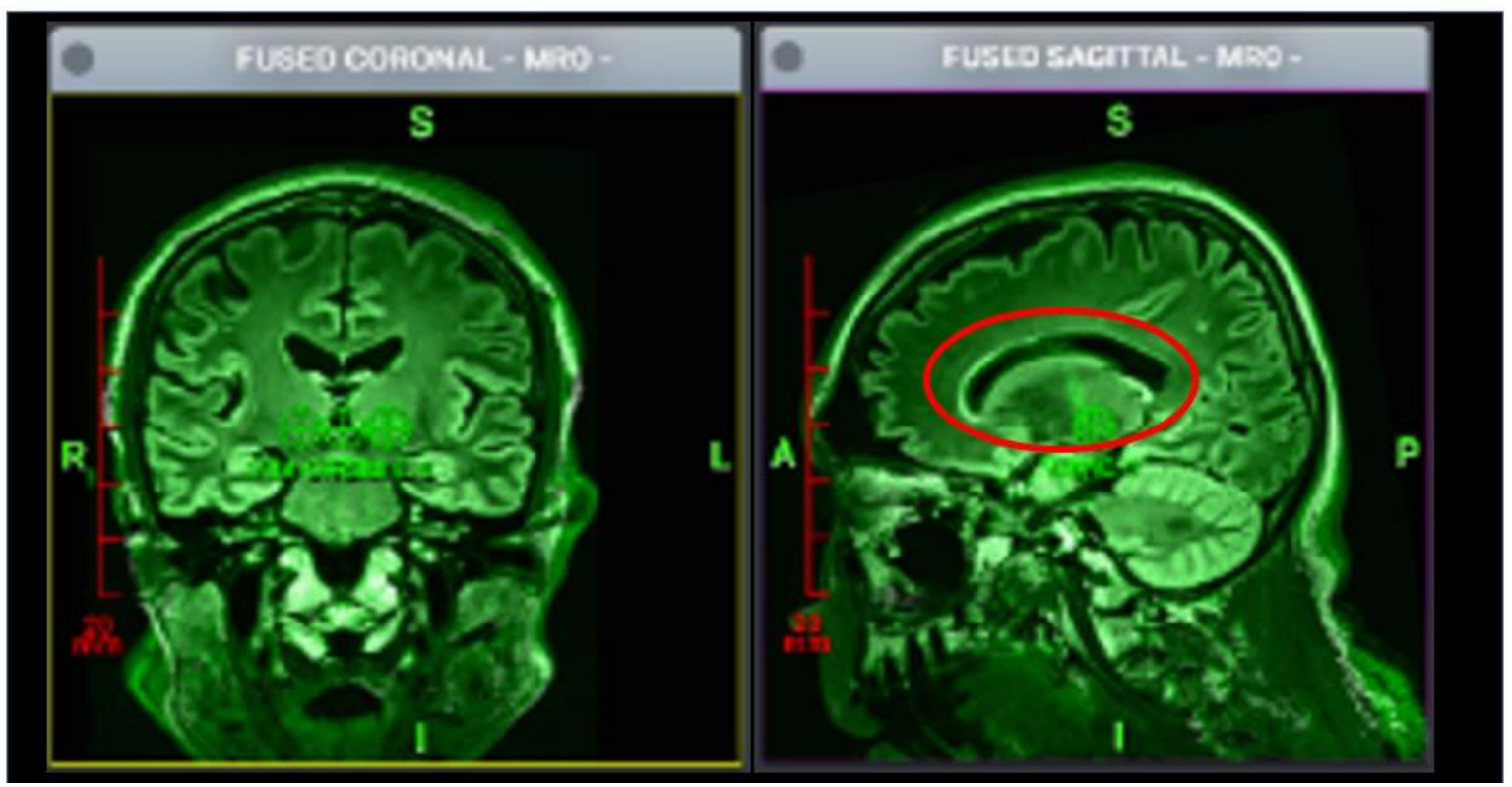

Figure 3. The example of Coronal and Axial Planes of Patients without Cyst (Patient 1).

\subsection{Method}

This paper proposes an automatic Vim location method. There are three steps in the proposed method: (1) define the anterior commissure (AC), posterior commissure (PC) and midline reference (MR) points in 3-vector coordinates; (2) redefine the plane passing through those points using 3 normal vectors that have been rotated by a quaternion; and (3) determine the Vim targeting location based on Coordinate-based targeting and Guiot's method.

\subsubsection{Define AC, $\mathrm{PC}$, and $\mathrm{MR}$}

The anterior commissure (AC), posterior commissure (PC), and midline (mid-sagittal plane) reference (MR) are used to align the brain [18]. The location of the $\mathrm{AC}$ is in the anterior wall of the third ventricle at the upper end of the lamina terminalis. The location of the PC is in the posterior wall of the third ventricle. These points can be determined based on the sagittal plane and the axial plane. An example of AC-PC is shown in Figure 4.

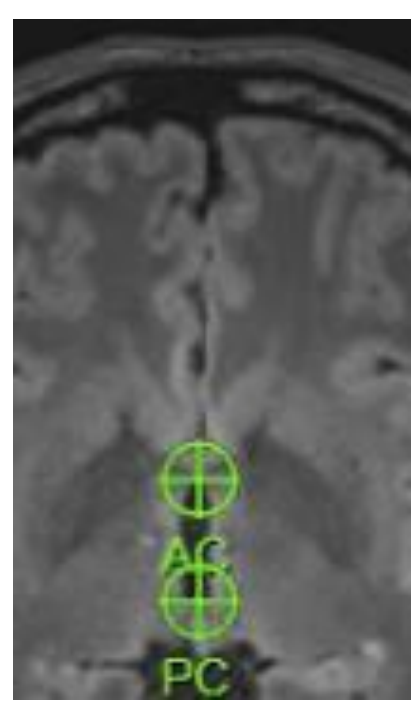

(a) Axial plane

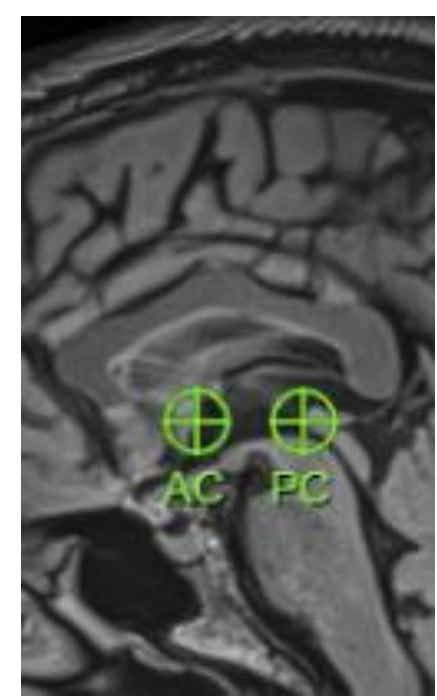

(b) Sagittal plane

Figure 4.AC-PC in the axial plane and the sagittal plane 
After determining the points of AC-PC, the midline (mid-sagittal plane) reference (MR) is determined. The mid-sagittal plane divides the left and the right brain. The point of MR is obtained based on a point in a line that is perpendicular to the AC-PC line and splits the brain stem. MR is denoted as M2 (the yellow circle) in Figure 5.

Based on the points of AC, PC, and MR, the plane for determining the Vim location is obtained (denoted as Plane A or Pa). A visualization of Pa is shown in Figure 6. The position of this plane is in the center of the brain. $\mathrm{C}$ in Figure 7 is the center point of the plane. Even though Pa has been obtained, the geometric calculation cannot be conducted on the plane; we must redefine Pa using 3 normal vectors that have been rotated by a quaternion.

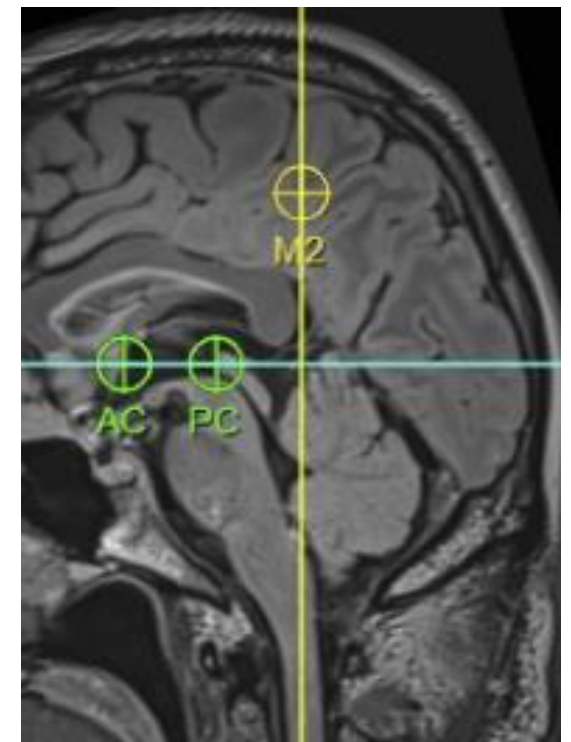

(a) Sagittal plane

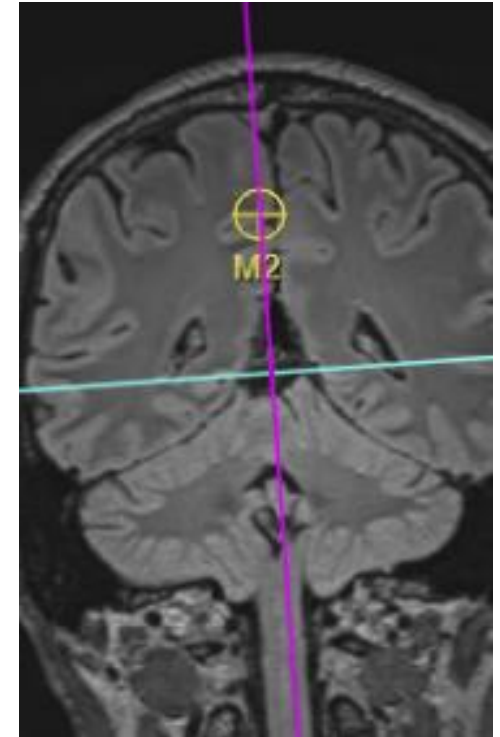

(b) Coronal plane

Figure 5.MR in the sagittal plane and the coronal plane

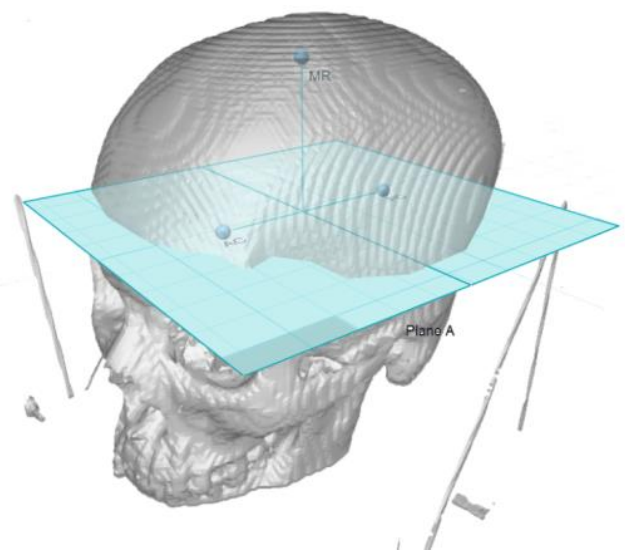

Figure 6. Visualization of a plane that is inside a head 


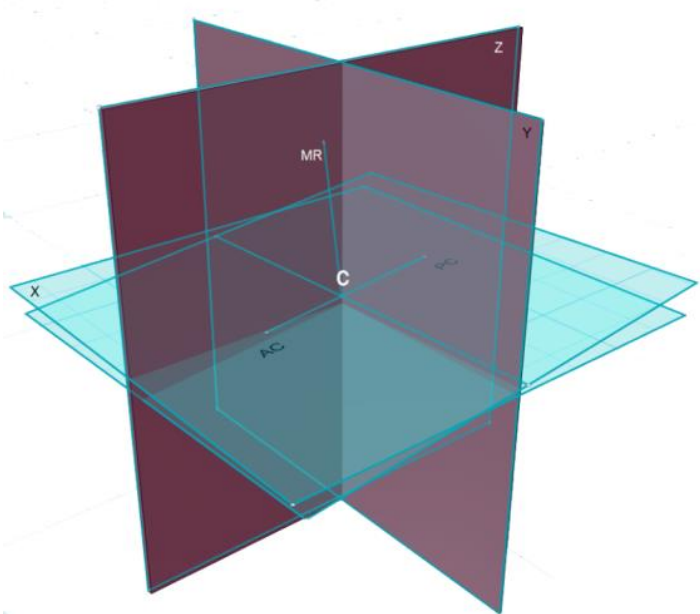

Figure 7. The position of Plane A between normal Plane X, Plane Y, and Plane Z.

\subsubsection{Define AC, PC, and MR}

The redefinition of the plane is explained step by step in Algorithm 1. The inputs are the points of $\mathrm{AC}, \mathrm{PC}, \mathrm{MR}$ and 3 normal vectors. The result is 3 normal vectors that have been rotated by a quaternion [19].

Algorithm 1: Redefine The Obtained Plane

Inputs: AC, PC, MR and 3 normal vectors that are determined by Equation (3)

Outputs: $\overrightarrow{N r_{x}}, \overrightarrow{N r_{y}}, \overrightarrow{N r_{z}}$

$1 \quad$ Calculate the quaternion $Q 1$ between $\overrightarrow{N r_{y}}$, and $\overrightarrow{A C P C}$ based on Equations (4) and (5)

2 Project AC $\left(T^{\prime}{ }^{\prime}{ }^{\prime}\right)$ and MR $\left(T^{\prime}{ }^{\prime}\right)$ to Py based on Equations (6) to (13) [19]

3 Calculate the angle of $T^{\prime}{ }_{A C}$ and $T^{\prime}{ }_{M R}$, then multiply it by $Q 1$ based on Equations (14) and (15) [19]

$4 \quad$ Rotate the normal vector using $Q 1$ based on Equations (16) and (18)

$$
\begin{aligned}
& \overrightarrow{N_{x}}=\left[\begin{array}{l}
1 \\
0 \\
0
\end{array}\right], \overrightarrow{N_{y}}=\left[\begin{array}{l}
0 \\
1 \\
0
\end{array}\right], \overrightarrow{N_{z}}=\left[\begin{array}{l}
0 \\
0 \\
1
\end{array}\right] \\
& \overline{A C P C_{N}}=\frac{\overline{A C P C}}{\sqrt{\left(A C_{x}-P C_{x}\right)^{2}+\left(A C_{y}-P C_{y}\right)^{2}+\left(A C_{z}-P C_{z}\right)^{2}}} \\
& Q 1=\text { QuatFromTwoVector }\left(\overrightarrow{N_{y}}, \overrightarrow{A C P C_{N}}\right)
\end{aligned}
$$

$$
\begin{gathered}
\text { QProject }=\text { QuatMul }\left(\text { Q1, QuaternionFromAxisAngle }\left(\overrightarrow{N_{x}},-90^{\circ}\right)\right) \\
\text { QProject }=\text { QuatMul }\left(\text { Q1, QuaternionFromAxisAngle }\left(\overrightarrow{N_{z}}, 180^{\circ}\right)\right) \\
\text { QProject }=\left[\begin{array}{l}
- \text { QProject }_{x} \\
- \text { QProject }_{y} \\
- \text { QProject }_{z}
\end{array}\right] \\
P=P C+\left(\frac{\text { distFromPC }}{\text { VoxelSpacing } \times \overline{N r_{x}}}\right)
\end{gathered}
$$

$$
\begin{aligned}
& T_{A C}=A C-C \\
& T_{M R}=M R-C
\end{aligned}
$$

\section{Research Square Preprint | CC BY 4.0}




$$
\begin{gathered}
T_{A C}^{\prime}=T_{A C}^{\prime} \times \text { ProjectMatrix, } \\
T^{\prime}{ }_{M R}=T^{\prime}{ }_{M R} \times \text { ProjectMatrix } \\
T_{\text {angle }}^{\prime}=\operatorname{atan} \times\left(\frac{T_{A C_{x}}^{\prime}-T^{\prime}{ }_{M R_{x}}}{T_{A C_{y}}^{\prime}-T^{\prime}{ }_{M R_{y}}}\right) \\
Q 1=\text { QuatMul }\left(Q 1, \text { QuaternionFromAxisAngle }\left(\overrightarrow{N y,-T_{\text {angle }}}\right)\right) \\
\overrightarrow{N r_{x}}=\text { QuatRotateVector }\left(Q 1, \overrightarrow{N_{x}}\right) \\
\overrightarrow{N r_{y}}=\text { QuatRotateVector }\left(Q 1, \overrightarrow{N_{y}}\right) \\
\overrightarrow{N r_{z}}=\text { QuatRotateVector }\left(Q 1, \overrightarrow{N_{z}}\right)
\end{gathered}
$$

\subsubsection{Determine Vim Location}

The last step is determining the Vim location. The automatic Vim localization based on Guiot's method is determined by Equations (19) to (21), while that based on Coordinate-based targeting is determined by Equations (22), (23), and (21).

$$
\begin{aligned}
& \text { distFromPC } \\
& =\frac{1}{4} \times \frac{\overrightarrow{A C P C}}{\sqrt{\left(A C_{x}-P C_{x}\right)^{2}+\left(A C_{y}-P C_{y}\right)^{2}+\left(A C_{z}-P C_{z}\right)^{2}}} \\
& P=P C+(\text { distFromPC } \times \overrightarrow{A C P C}) \\
& V I M=P \pm \frac{15}{\text { VoxelSpacing } \times \overrightarrow{N r}_{x}} \\
& \text { distFromPC }=\operatorname{rand}(6,7) \\
& P=P C+\left(\frac{\text { distFrom } P C}{\text { VoxelSpacing } \times \overline{N r_{x}}}\right)
\end{aligned}
$$

\section{RESULTS AND ANALYSIS}

Firstly, the proposed application was compared with the existing neurosurgery planning application Inomed Planning Software (IPS). The comparison is shown in Figure 8. The locations of the Vim, AC, and PC were the same, so the point determination method of the proposed application was proved to be correct.

Secondly, we compared the accuracy and robustness of the results of Coordinate-based targeting and Guiot's methods. The accuracy is obtained by similarity percentages between the results of the methods and the ground truth by using Euclidean Distance. The robustness is obtained by average error results in each slope based on X plane, Y plane, and Z plane. Based on the similarity percentages in Figure 9, there is same results on two patients, the higher similarity of results determined by Coordinate-based targeting (C) on five patients, and higher similarity of result determined by Guiot's (G) on four patients. It can be concluded that Coordinate-based targeting has higher accuracy than Guiot's. Figure 9 shows the lowest similarity percentages of both methods is on $2^{\text {nd }}$ Patient who got a cyst. It proves that an inaccuracy of CC shape influences the accuracy of Vim locations.

Thirdly, we determined the robustness of Vim localization methods, i.e. Coordinate-based targeting and Guiot's. The smaller error results in each slope of medical images, the higher robustness of the method. Figure 10 shows that Coordinate-based targeting has the smallest average of error results on mostly slope of medical images. However, Guiot's has the smallest average of error results if the slope towards X-plane. It can be concluded that Coordinate-based targeting is more robust then Guiot's. 


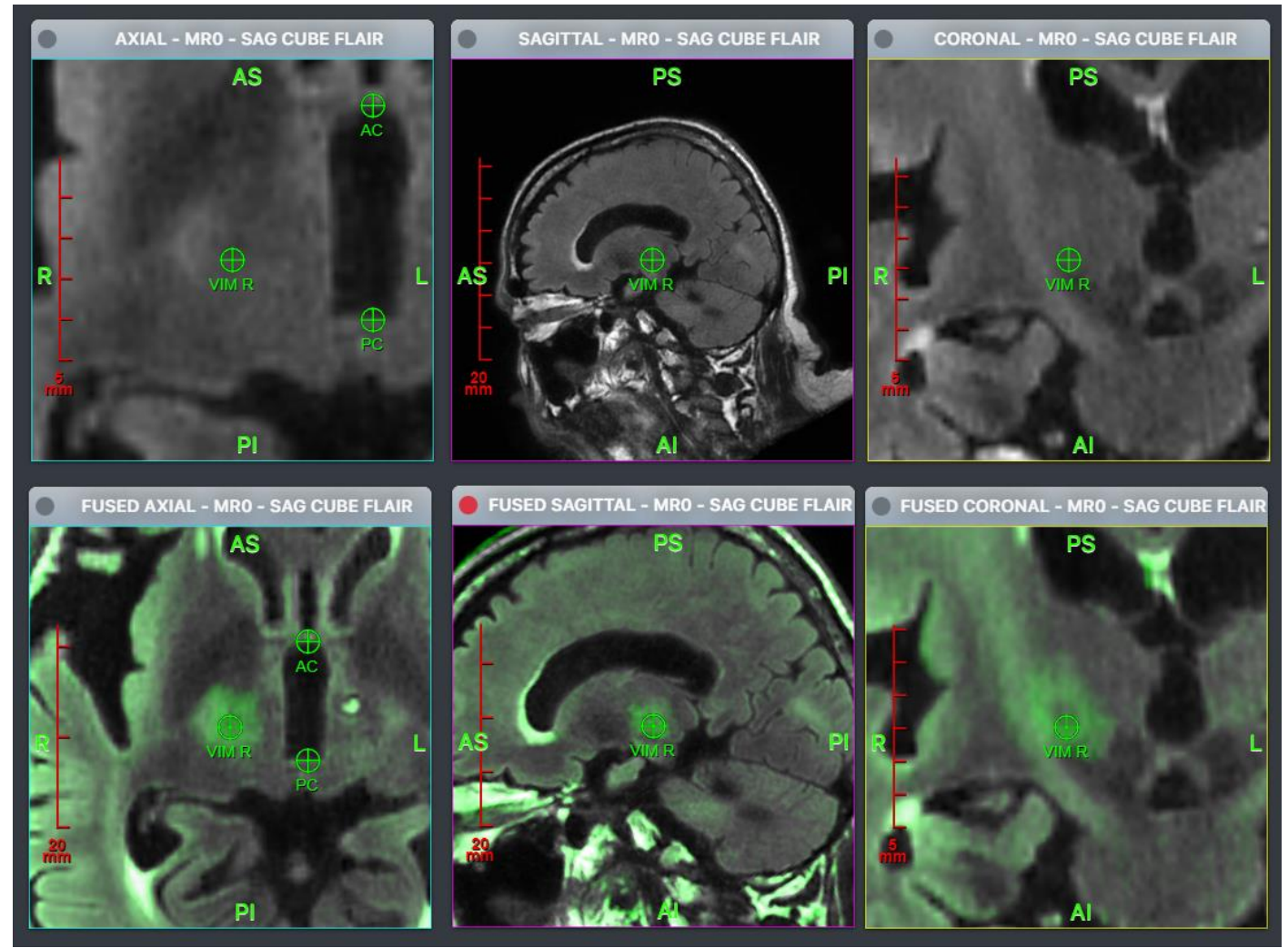

Figure 8. Comparison between the proposed application (above) and the existing neurosurgery application (below).

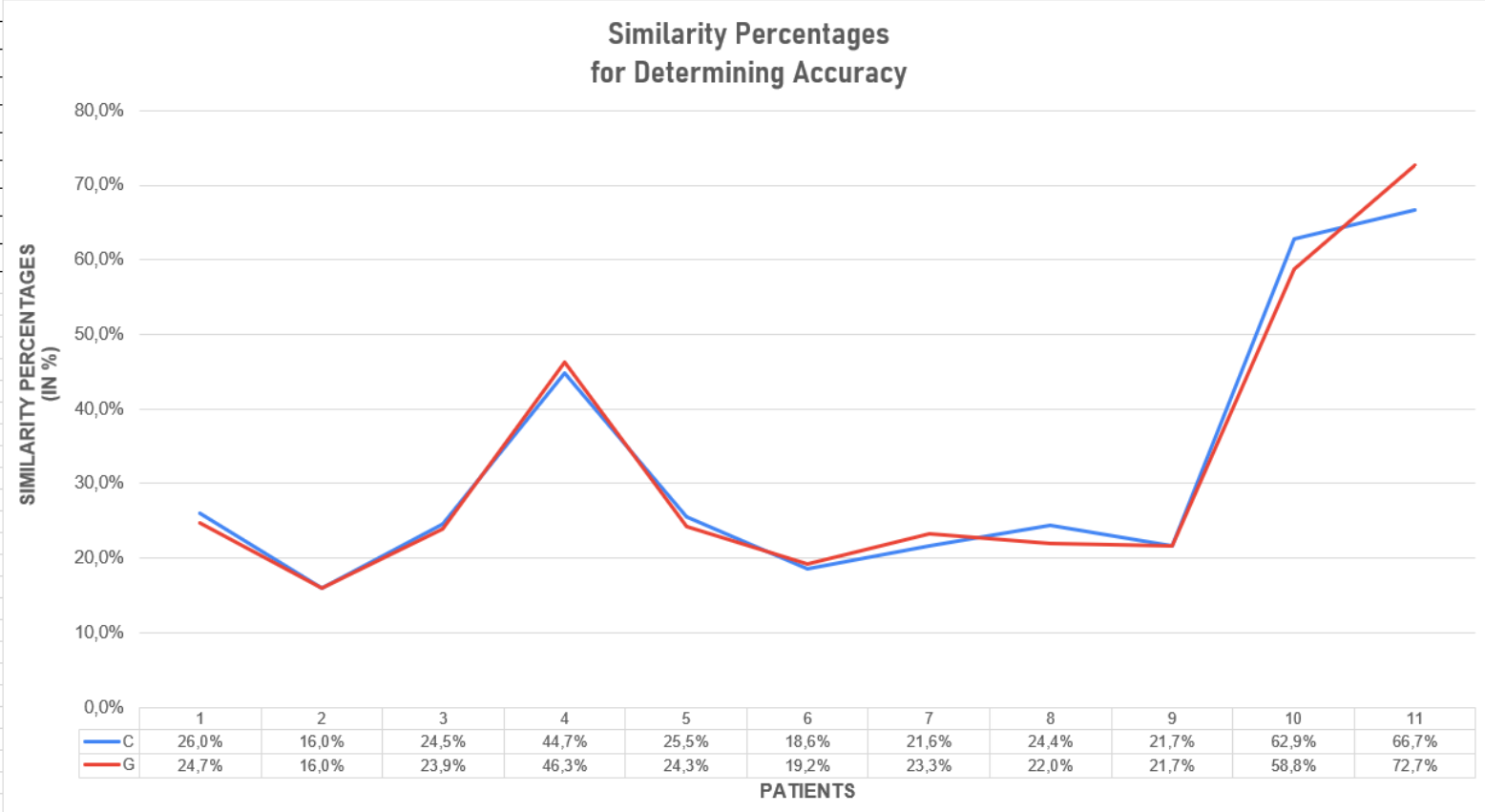

Figure 9. Similarity Percentages for Determining Accuracy (C: Coordinate-based targeting, G: Guiot's) 


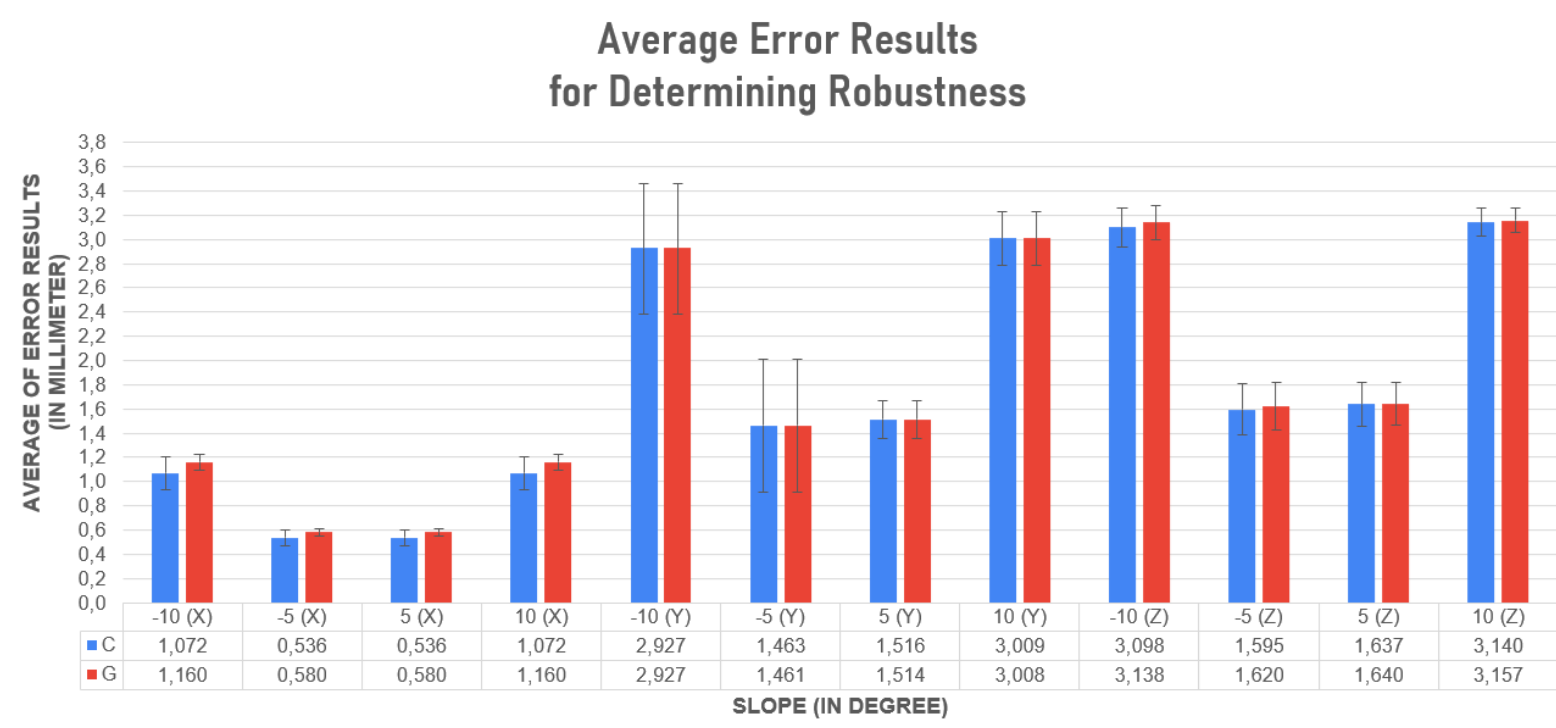

Figure 10. Average of Error Results for Determining Robustness (C: Coordinate-based targeting, G: Guiot's)

\section{CONCLUSION}

This paper proposed a method for automatic VIM localization based on Coordinate-based targeting and Guiot's method. There are several steps in the proposed method. The first step is determining the plane of the anterior commissure (AC) and the posterior commissure (PC) because this paper presents the centre of the MRI image as the middle point of the AC-PC plane. Then, the proposed automatic method converts the coordinates from millimetres to voxels to determine the Vim location. This is because Coordinate-based targeting and Guiot's method measure the Vim location in millimetres while three-dimensional images are measured in voxels.

This paper determines the correctness of proposed automatic Vim localization methods by comparing the proposed application and the existing application, Inomed Planning Software (IPS). By using the same method, the Vim location is same in both of those applications. It shows that the method of automatic Vim localization is correct. Then, this paper also measures the accuracy and the robustness of Coordinate-based targeting method and Guiot's method. The result shows that Coordinate-based targeting has higher accuracy and more robust than Guiot's.

\section{Abbreviations}

AC: Anterior Commissure; CC: Corpus Callosum; CT: Computerized Tractography; MR: Midline Reference; MRI: Magnetic Resonance Imaging; PC: Posterior Commissure; Vim: Ventral Intermediate Nucleus.

\section{Availability of data and materials}

The used raw dataset in this research is not publicly available. Readers can contact the author if they want to access the data

\section{Competing interest}

The authors declare that they have no competing interests.

\section{Funding}

This research did not receive any specific grant from funding agencies in the public, commercial, or not-for-profit sectors.

\section{Authors' contributions}

RS is the supervision that has the idea for creating automatic Vim localization. KRS composed the manuscript and did the experiment. MAR built the program. AF is neurosurgeon that operated on patients with Parkinson's disease and determine the used methods. AT and AHB are neurosurgeon that evaluates the results that are obtained by KRS. All authors read and approved the final manuscript. 


\section{Acknowledgements}

The authors would like to express their sincere thanks to Institut Teknologi Sepuluh Nopember, Layanan Beasiswa dan Pendanaan Riset Indonesia (LPDP) and General Hospital Dr. Soetomo Surabaya.

\section{REFERENCES}

1. Poewe W, Seppi K, Tanner C, et al (2017) Nature Reviews Disease Primers. Nature Reviews Disease Primers 3:. https://doi.org/10.1038/nrdp.2017.101

2. Nikkhah G, Carvalho GA, Pinsker M (2013) Functional Neurosurgery in Parkinson's Disease: A Long Journey from Destruction Over Modulation Towards Restoration. In: Nikkhah G, Pinsker M (eds) Stereotactic and Functional Neurosurgery. Springer Vienna, Vienna, pp 5-11

3. Sidiropoulos C, Mubita L, Krstevska S, Schwalb JM (2015) Successful Vim targeting for mixed essential and parkinsonian tremor using intraoperative MRI. Journal of the neurological sciences 358:488-489. https://doi.org/10.1016/j.jns.2015.08.1553

4. Sammartino F, Krishna V, King NKK, et al (2016) Tractography-Based Ventral Intermediate Nucleus Targeting: Novel Methodology and Intraoperative Validation. Movement Disorders 31:1217-1225. https://doi.org/10.1002/mds.26633

5. Sarrigiannis PG, Zhao Y, He F, et al (2015) Direct functional connectivity between the thalamus (Vim) and the contralateral motor cortex: Just a single case observation or a common pathway in the human brain? Brain Stimulation 8:1230-1233. https://doi.org/10.1016/j.brs.2015.08.006

6. Ewert S, Plettig P, Li N, et al (2018) Toward defining deep brain stimulation targets in MNI space: A subcortical atlas based on multimodal MRI, histology and structural connectivity. NeuroImage 170:271282. https://doi.org/10.1016/j.neuroimage.2017.05.015

7. Rodriguez-Oroz MC, Moro E, Krack P (2012) Long-term outcomes of surgical therapies for Parkinson's disease. Movement Disorders 27:1718-1728. https://doi.org/10.1002/mds.25214

8. Taira T, Horisawa S, Takeda N, Ghate P (2018) Stereotactic Radiofrequency Lesioning for Movement Disorders. Progress in Neurological Surgery 33:107-119. https://doi.org/10.1159/000481079

9. Khaled M. Krisht, Mohammad Sorour, Martin Cote, et al (2015) Marching beyond the sella: Gerard Guiot and his contributions to neurosurgery. Journal of Neurosurgery 122:464-472. https://doi.org/10.3171/2014.10.JNS131919

10. Guiot G, Arfel G, Derome P, Kahn A (1968) [Neurophysiologic control procedures for sterotaxic thalamotomy]. Neuro-Chirurgie 14:553-566

11. Jakab A, Blanc R, Berényi EL, Székely G (2012) Generation of individualized thalamus target maps by using statistical shape models and thalamocortical tractography. American Journal of Neuroradiology 33:2110-2116. https://doi.org/10.3174/ajnr.A3140

12. Jakobs M, Krasniqi E, Kloß M, et al (2018) Intraoperative stereotactic MRI for deep brain stimulation electrode planning in patients with movement disorders. World Neurosurgery 1-8. https://doi.org/10.1016/j.wneu.2018.07.270

13. Tamura M, Hayashi M, Konishi Y, et al (2013) Advanced Image Coregistration within the Leksell Workstation for the Planning of Glioma Surgery: Initial Experience. Journal of Neurological Surgery Reports 74:118-122. https://doi.org/10.1055/s-0033-1358380

14. Hashizume A, Akimitsu T, Iida K, et al (2016) Novel software for performing leksell stereotactic surgery without the use of printing films: Technical note. Neurologia Medico-Chirurgica 56:193-197. https://doi.org/10.2176/nmc.tn.2015-0155

15. Sutter M, Eggspuehler A, Muller A, Dvorak J (2007) Multimodal intraoperative monitoring: An overview and proposal of methodology based on 1,017 cases. European Spine Journal 16:. https://doi.org/10.1007/s00586-007-0417-8

16. inomed Medizintechnik Inomed Planning - Software IPS. https://www.en.inomed.com/products/functional-neurosurgery/ips/

17. Eisinger RS, Wong J, Almeida L, et al (2017) Ventral Intermediate Nucleus Versus Zona Incerta Region Deep Brain Stimulation in Essential Tremor. Movement Disorders Clinical Practice 5:75-82. https://doi.org/10.1002/mdc3.12565

18. Bazin P, Sciences B, Gandler W, et al (2005) Free software tools for atlas-based volumetric neuroimage analysis Free Software Tools for Atlas-based Volumetric Neuroimage Analysis. https://doi.org/10.1117/12.595602

19. Bar-Itzhack IY (2000) New method for extracting the quaternion from a rotation matrix. AIAA Journal of Guidance, Control and Dynamics 23:1085-1087 